\title{
Evaluating the rheological property of Irvingia gabonensis and Abelmoschus esculentus as a substitute to conventional Pac- $R$ on cutting carrying capacity and hole cleaning
}

\author{
K. K. Ihekoronye ${ }^{1}$ (D) N. C. Izuwa ${ }^{1} \cdot$ N. U. Okereke ${ }^{1} \cdot$ S. T. Ekwueme ${ }^{1} \cdot$ R. C. Amaefula ${ }^{1}$
}

Received: 18 July 2019 / Accepted: 27 October 2019 / Published online: 7 November 2019

(c) The Author(s) 2019

\begin{abstract}
In this work, experiment was carried out resulting in the utilization of local viscosifiers as substitute to conventional method (PAC-R) for hole cleaning purposes in drilling mud. The proposed viscosifiers are Irvingia gabonensis (Ogbono) and Abelmoschus esculentus (Okro) as alternative to the imported poly-anionic cellulose-regular (PAC-R) which is used in cutting carrying capacity efficiency. Laboratory tests were carried out on the proposed viscosifiers to evaluate their rheological properties. Slip velocity, annular velocity, shear stress, shear rate and cuttings transport efficiency on hole cleaning parameters were calculated to ascertain the effectiveness of the proposed viscosifiers in comparison with the conventional one (PAC-R). The results of the study showed that the proposed viscosifiers Sample B Irvingia gabonensis (Ogbono) had cutting carrying capacity of $96 \%$ for 5, 8, 10 and 15 (g), respectively. However, Sample C Abelmoschus esculentus (Okro) had 96\% for $5 \mathrm{~g}$ and $8 \mathrm{~g}$ and $95 \%$ for $10 \mathrm{~g}$ and $15 \mathrm{~g}$ on the cutting transport efficiency. Based on the result of this work, the proposed viscosifiers compared favorably to that of PAC-R on hole cleaning and cutting carrying capacity.
\end{abstract}

Keywords Rheology · Cutting carrying capacity · Irvingia gabonensis · Abelmoschus esculentus · Transport efficiency

\section{List of symbols}

RPM Revolution per minute

$n \quad$ Flow behavior index

$P_{\mathrm{v}} \quad$ Plastic viscosity

$Y_{\mathrm{p}} \quad$ Yield point

$K \quad$ Consistency factor

$Y \quad$ Shear stress

$y \quad$ Shear rate

$\mathrm{Vt} \quad$ Net velocity of the cutting

$T_{\text {eff }} \quad$ Transport efficiency
$V_{\text {a }} \quad$ Annular velocity

$d \quad$ Cutting diameter

$Q \quad$ Flow rate

dh Hole diameter

dp Outer diameter of drill pipe

$V_{\mathrm{s}} \quad$ Slip velocity

$U_{\text {eff }} \quad$ Effective viscosity

$P_{p} \quad$ Cutting density

$\rho_{f} \quad$ Mud weight

PAC-R Poly-anionic cellulose-regular
K. K. Ihekoronye

kelechiking4@gmail.com

N. C. Izuwa

ncizuwa@yahoo.com

N. U. Okereke

okerekenu79@gmail.com

S. T. Ekwueme

stanleyekwueme@yahoo.com

R. C. Amaefula

ramseyamaes@gmail.com

1 Petroleum Engineering, Federal University of Technology Owerri, Owerri, Nigeria

\section{Introduction}

Drilling fluids play a crucial role in carrying out the drilled cuttings and to lubricate the bits for fast and effective penetration into the formation. Cuttings removal is a function of the mud rheology, wellbore size and the subsurface conditions prevailing while drilling (Ozbayoglu 2007; Igwilo and Zaka 2014). The rheological properties of the drilling mud must be suitable to perform the functions of transporting drilled cuttings from the bottom of the well to the surface, keeping the drill bits, cooling the drill string, holding cuttings in suspension when mud circulation ceases 
and preventing formation fluids from gaining access into the wellbore (Blkoor and Fattah 2013). Drilling mud must have high cutting carrying capacity and hole cleaning capability (Baroid 2006). The desired rheology includes high shear thinning viscosity with a high ratio of yield point to plastic viscosity and relatively high gel strength (Belavadi and Chukwu 1994). Bazarnova et al. (2001) in their work observed that polymers like xanthan gum and carboxyl methyl cellulose (CMC) have been used successfully with bentonite clay to achieve good carrying capacity in waterbased mud (WBM). Izuwa (2015) observed that it was necessary to evaluate the cutting carrying ability of these new viscosifiers. In addition, Zhang et al. (2012) reported that adequate hole cleaning is important in the drilling industry to eschew the operational problems that arise if sufficient wellbore cleaning is not achieved. Poor hole cleaning challenges include excessive pull on trips, hole pack-off, excessive equivalent circulating density (ECD), formation break down, slow rate of penetration and difficulty in removing casing (Dosunmu et al. 2012). The difficulty in removing cuttings is caused by the interaction of the drilling mud with the cuttings bed to form cutting bed gel (Noah 2013).

\section{Literature review}

Drilling fluid composition is designed to minimize gel formation in the cuttings bed (Igwilo et al. 2016). However, one of the functions of the drilling mud is to remove the cuttings generated by the drill string (Unegbu 2010). In addition, the drilling fluid properties should be optimized to ensure a sufficient shear stress on the cuttings for adequate cuttings removal (Doan et al. 2000). The cutting carrying capacity of a drilling fluid is its ability to transport the cuttings up the casing-pipe annulus to the surface (Ogunrinde and Dosunmu 2012). Moreover, poor wellbore cleaning can result to operational challenges which may lead to reduction in revenue derived because of non-productive time and higher operating costs (Dosunmu et al. 2012). Mozaffari et al. (2015) observed that asphaltenes and viscosity of heptol (80:20) and heptanes diluted bitumen was importance for improving heavy oil extraction of Athabasca bitumen.

Sifferman and Becker (1990) observed that cutting transport efficiency can increase as fluid viscosity increases. Their work further revealed that more cuttings are transported in a laminar flow for about (85-90\%), while in a turbulent flow, the cuttings removal is about 75\%. Doan et al. (2000) reported in their experiment conducted with different cutting sizes, showed that smaller cuttings are more difficult to be removed than larger cuttings in water-based fluid. Suspension of the drill cutting in the drilling mud is favored by the rotation of drill pipe especially when drilling is not in progress (Ozbayoglu 2007; Belavadi and Chukwu 1994; Sifferman and Becker
1990; Walker and Li 2001). Evelyn et al. (2019) observed that green materials (Averrhoa carambola) can be a possible substitute for PAC-R in water-based drilling system. The result of their study showed that Kian (Averrhoa carambola) has the characteristic of PAC-R in API standard and as viscosifiers.

Kerunwa and Gbaranbiri (2018) proposed local viscosifiers obtained from Mucuna flagellipe (Ukpo), Brachystegia eurycoma (Achi), Afzelia africana (Akpalata) and Detarium microcarpum (Ofor) as a substitute for imported viscosifiers (PAC-R) used as a drilling fluid additives. They investigated that Mucuna flagellipe (Ukpo) had a better viscosity compared to Achi, Akpalata and Ofor of the same concentration. In addition, Hossain and Wajheeuddin (2016) evaluated the effect of annular fluid velocity and yield point on cutting carrying capacity of drilling fluids. They noted that annular fluid velocity and yield point had positive influence on cutting transport. The cutting transport efficiency was computed from the equation given below by Baroid (1998, 2006), Igwilo and Zaka (2014).

$T_{\mathrm{E}}=\left(\frac{V_{\mathrm{a}}-V_{\text {slip }}}{V_{\mathrm{a}}}\right) \times 100$

The Herschel Buckley model is a modified power law model (Baroid 1998; Duru et al. 2005; Igwilo and Zaka 2014). Igwilo and Zaka (2014) and Izuwa (2015) reported that the effectiveness of drilling fluid is measured based on its rheological properties which include yield point, shear rate, shear stress and plastic viscosity. The functions of the drilling fluids are dependent on cuttings transportation along the wellbore. Baroid (1998) reported that clay material in water-based mud is responsible for an increase in viscosity which improves the lifting capacity of the mud to carry cuttings to the surface and building a thin wall cake in permeable zones, thus preventing fluid loss.

The study focuses on using power law model and modified power law model to evaluate the rheological properties of the local viscosifiers and the cuttings carrying capacity of the proposed mud.

\section{Objective of the study}

(i) Investigation of the rheological property of Irvingia gabonensis and Abelmoschus esculentus of the local viscosifiers.

(ii) Determination of the cutting carrying capacity of the local additives using power law model and modified power law model

(iii) Evaluation of the local additives to determine its hole cleaning capacity which is a key factor in drilling mud. 
(iv) Evaluation of the transport efficiency of the local additives in comparison with the conventional approach (poly-anionic cellulose-regular)

\section{Importance of the study}

(i) The local agro materials Irvingia gabonensis and Abelmoschus esculentus can be used as a hole cleaning agent in drilling operations.

(ii) The result from the study showed that the proposed viscosifiers can be effective in carrying drilled cutting to the surface.

(iii) Drilling cost will be minimized because these local additives are readily available in the market when compared to the cost of importing foreign ones.

(iv) The proposed additives could serve as an alternative to conventional poly-anionic cellulose-regular for transport efficiency of the drilled cutting.

\section{Methodology}

\section{Collection of the samples}

The samples used in the study were gotten from Owerri Cluster market, Imo state, Nigeria (Figs. 1, 2).

\section{Equipments used in the research work}

(i) Retort stand (ii) beakers (iii) filter paper (iv) HPHT filter press (v) weighing balance (vi) stopwatch (vii) measuring cylinder (viii) electric grinder (ix) oven (x) mud balance.

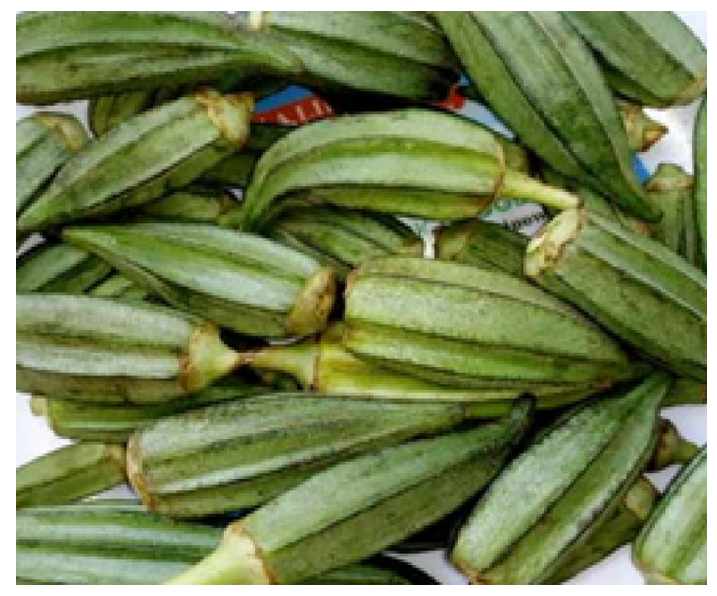

Fig. 1 Sample of Abelmoschus esculentus

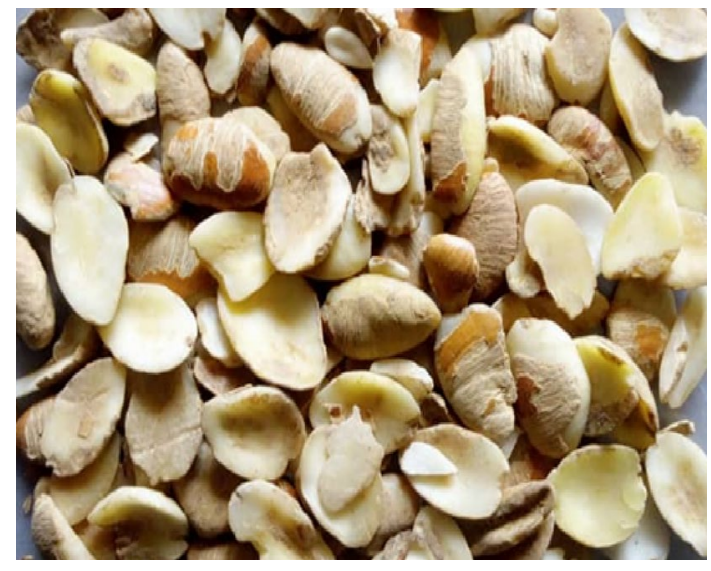

Fig. 2 Sample of Irvingia gabonensis

\section{Sample preparation}

The samples were properly washed to avoid bacterial contaminate. It was later stored in a basket. The Sample B was sun-dried for one week. Sample B was grinded to remove moisture for easy oil extraction from the solute as shown in Fig. 3. Sample C was sun-dried for three days to remove water. The dried Sample C was later grinded to get a fine microparticle (130 micros) and then stored in a plastic bag at room temperature.

\section{Experimental conditions}

(i) The experiment was conducted under atmospheric condition.

(ii) The temperature range was $150-250{ }^{\circ} \mathrm{F}$.

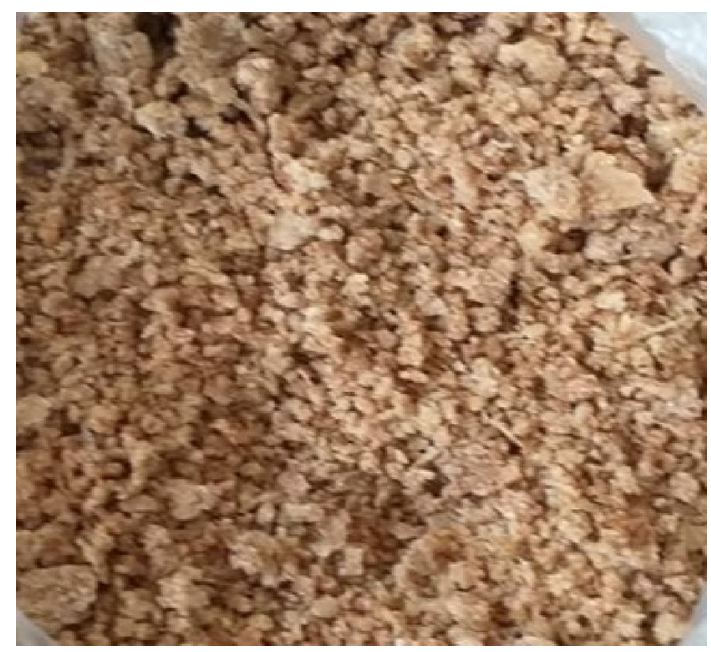

Fig. 3 Grounded sample of Irvingia gabonensis

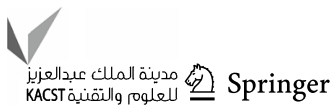




\section{Experimental procedure}

Sample C, Abelmoschus esculentus (Okro), was sun-dried and grinded to a smallest particle for uniformity. It was introduced into a solution (that is mixing water with the Okro) to ascertain its viscosity at different grams. In addition, Sample B (Irvingia gabonensis) was collected to an electric source after moisture removal (sun-dried). Sample B was wrapped with a filter paper to extract the oil from the solute. It was later placed in a thimble of a Soxhlet extractor. The Soxhlet extractor has three major components: (1) a percolator which helped in circulating the solvent, (2) a thimble which retains the solid to be laved and (3) a siphon which empties the thimble. Hexane solvent was poured to the connected Soxhlet extractor and put in a water bath to heat up. The solvent was heated to reflux. The solvent vapor moved up a distillation arm into the chamber where the thimble solid was housed. The condenser helped to cool the solvent vapor. However, when the Soxhlet chamber gets filled, the chamber was emptied by the siphon. More so, hexane evaporates and soaked up the oil and returned back to the distillation flask and was separated by rotary evaporator. Figure 4 shows the schematic diagram of Soxhlet extractor, while Fig. 5 is the experimental setup

\section{Experimental test for the water-based mud}

Drilled water of $300 \mathrm{ml}$ was measured using a measuring cylinder at different beakers. The water was then treated with alum to remove water hardness. Fifteen grams of bentonite was added to the treated water for $30 \mathrm{~min} .3 .0 \mathrm{~g}$ of poly-anionic cellulose-regular (Pac-R) was added and mixed thoroughly for about $10 \mathrm{~min}$ before barite $70 \mathrm{~g}$ was

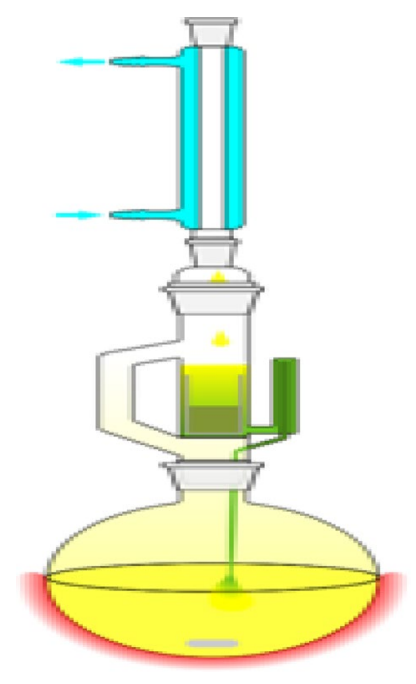

Fig. 4 Diagram of Soxhlet extraction

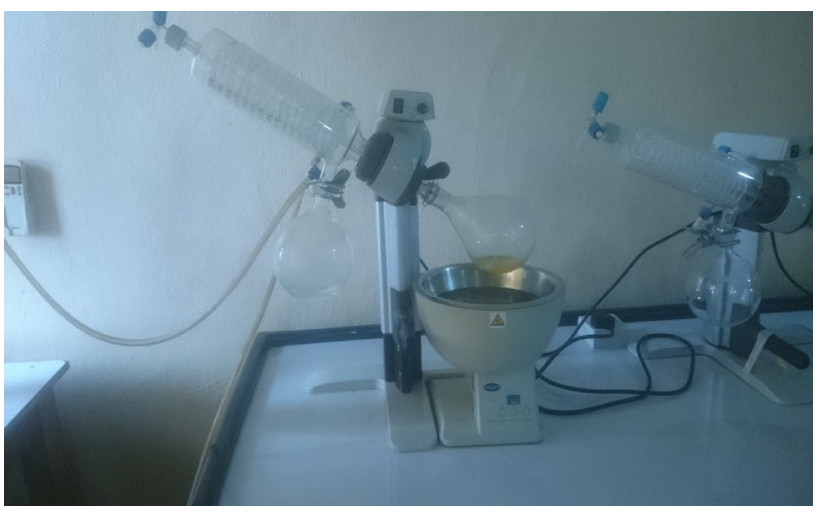

Fig. 5 Experimental setup

added for $30 \mathrm{~min}$ and mixed thoroughly. The above procedure was repeated for all the samples (A, B and C) used in the experiment (Table 1).

\section{Rheology test}

In the rheology test, the performance of the local viscosifier was compared with the standard water-based mud formulation. The viscometer was calibrated before taking the rheological properties of the formulated water-based mud. The mud sample was heated to $250^{\circ} \mathrm{F}$ using a thermo-cup. At the attainment of $250{ }^{\circ} \mathrm{F}$, the rheological parameters were taken by placing the viscometer nub on 600,300 , 200, 100, 60 and $30 \mathrm{rpm}$, respectively, while the dial reading was also taken at intervals using the different mud samples (Tables 2, 3, 4, 5, 6, 7, 8).

\section{Field data used for the experimental work}

The field data used in this study were gotten from the Niger Delta oil field (Table 9).

Table 1 Water-based formulation

\begin{tabular}{lccc}
\hline Components & $\begin{array}{l}\text { Control } \\
\text { Sample A }\end{array}$ & Sample B & Sample C \\
\hline Water (ml) & 300 & 300 & 300 \\
Bentonite (g) & 15 & 15 & 15 \\
Caustic soda (g) & 0.4 & 0.4 & 0.4 \\
Soda ash (g) & 0.4 & 0.4 & 0.4 \\
PAC-R (g) & 3.0 & 3.0 & 3.0 \\
Barite (g) & 70 & 70 & 70 \\
Weighted mud weight (ppg) & 10.0 & 10.0 & 10.0 \\
\hline
\end{tabular}


Table 2 Rheology test at $5 \mathrm{~g}$

\begin{tabular}{llll}
\hline R.P.M & $\begin{array}{l}\text { Sample A (con- } \\
\text { trol experiment) }\end{array}$ & $\begin{array}{l}\text { Sample B } \\
\text { (Irvingia gabon- } \\
\text { ensis) }\end{array}$ & $\begin{array}{l}\text { Sample C (Abel- } \\
\text { moschus esculen- } \\
\text { tus) }\end{array}$ \\
\hline 600 & 63.0 & 63.0 & 62.0 \\
300 & 35.0 & 36.0 & 34.0 \\
200 & 29.0 & 28.0 & 29.0 \\
100 & 22.0 & 22.0 & 21.0 \\
60 & 17.0 & 16.0 & 17.0 \\
30 & 15.0 & 14.0 & 14.5 \\
\hline
\end{tabular}

Table 3 Rheology test at $8 \mathrm{~g}$

\begin{tabular}{llll}
\hline R.P.M & $\begin{array}{l}\text { Sample A (con- } \\
\text { trol experiment) }\end{array}$ & $\begin{array}{l}\text { Sample B } \\
\text { (Irvingia gabon- } \\
\text { ensis) }\end{array}$ & $\begin{array}{l}\text { Sample C (Abel- } \\
\text { moschus esculen- } \\
\text { tus) }\end{array}$ \\
\hline 600 & 66.0 & 67.0 & 62.5 \\
300 & 37.0 & 38.0 & 36.0 \\
200 & 30.5 & 30.0 & 29.5 \\
100 & 25.0 & 25.0 & 24.5 \\
60 & 20.0 & 21.0 & 21.0 \\
30 & 18.0 & 18.0 & 17.0 \\
\hline
\end{tabular}

Table 4 Rheology test at $10 \mathrm{~g}$

\begin{tabular}{llll}
\hline R.P.M & $\begin{array}{l}\text { Sample A (con- } \\
\text { trol experiment) }\end{array}$ & $\begin{array}{l}\text { Sample B } \\
\text { (Irvingia gabon- } \\
\text { ensis) }\end{array}$ & $\begin{array}{l}\text { Sample C (Abel- } \\
\text { moschus esculen- } \\
\text { tus) }\end{array}$ \\
\hline 600 & 64.0 & 62.0 & 66.0 \\
300 & 37.0 & 36.0 & 39.0 \\
200 & 31.0 & 32.0 & 31.0 \\
100 & 27.0 & 27.5 & 27.5 \\
60 & 22.5 & 22.5 & 22.0 \\
30 & 20.0 & 20.0 & 20.5 \\
\hline
\end{tabular}

Table 5 Rheology test at $15 \mathrm{~g}$

\begin{tabular}{llll}
\hline R.P.M & $\begin{array}{l}\text { Sample A (con- } \\
\text { trol experiment) }\end{array}$ & $\begin{array}{l}\text { Sample B } \\
\text { (Irvingia gabon- } \\
\text { ensis) }\end{array}$ & $\begin{array}{l}\text { Sample C (Abel- } \\
\text { moschus esculen- } \\
\text { tus) }\end{array}$ \\
\hline 600 & 63.0 & 62.0 & 61.0 \\
300 & 38.0 & 36.0 & 37.0 \\
200 & 32.0 & 32.5 & 32.0 \\
100 & 28.0 & 28.0 & 27.5 \\
60 & 24.5 & 24.0 & 24.0 \\
30 & 22.5 & 21.5 & 21.0 \\
\hline
\end{tabular}

Table 6 Water-based mud at a temperature of $150^{\circ} \mathrm{F}$

\begin{tabular}{llll}
\hline R.P.M & $\begin{array}{l}\text { Sample A (con- } \\
\text { trol experiment) }\end{array}$ & $\begin{array}{l}\text { Sample B } \\
\text { (Irvingia gabon- } \\
\text { ensis) }\end{array}$ & $\begin{array}{l}\text { Sample C (Abel- } \\
\text { moschus esculen- } \\
\text { tus) }\end{array}$ \\
\hline 600 & 53 & 54 & 56 \\
300 & 52 & 46 & 52 \\
200 & 38 & 37 & 37.5 \\
100 & 41 & 38 & 40 \\
60 & 42 & 40 & 42 \\
30 & 38 & 37 & 38 \\
\hline
\end{tabular}

Table 7 Water-based mud at a temperature of $200^{\circ} \mathrm{F}$

\begin{tabular}{llll}
\hline R.P.M & $\begin{array}{l}\text { Sample A (con- } \\
\text { trol experiment) }\end{array}$ & $\begin{array}{l}\text { Sample B } \\
\text { (Irvingia gabon- } \\
\text { ensis) }\end{array}$ & $\begin{array}{l}\text { Sample C (Abel- } \\
\text { moschus esculen- } \\
\text { tus) }\end{array}$ \\
\hline 600 & 47 & 46 & 45 \\
300 & 44 & 42 & 42 \\
200 & 38 & 37 & 37 \\
100 & 36 & 35 & 36 \\
60 & 34 & 33 & 34 \\
30 & 30 & 30 & 31 \\
\hline
\end{tabular}

Table 8 Water-based mud at a temperature of $250^{\circ} \mathrm{F}$

\begin{tabular}{llll}
\hline R.P.M & $\begin{array}{l}\text { Sample A (con- } \\
\text { trol experiment) }\end{array}$ & $\begin{array}{l}\text { Sample B } \\
\text { (Irvingia gabon- } \\
\text { ensis) }\end{array}$ & $\begin{array}{l}\text { Sample C (Abel- } \\
\text { moschus esculen- } \\
\text { tus) }\end{array}$ \\
\hline 600 & 45 & 43 & 44 \\
300 & 42 & 41 & 40 \\
200 & 38 & 37 & 37 \\
100 & 36 & 35 & 35 \\
60 & 33 & 31 & 30 \\
30 & 30 & 30 & 28 \\
\hline
\end{tabular}

Table 9 Field data

\begin{tabular}{lll}
\hline Parameter & Value & Unit \\
\hline Hole size diameter & 16.0 & Inch \\
Drill pipe ID & 4.50 & Inch \\
Drill pipe OD & 5.00 & Inch \\
Pump flow rate $(Q)$ & 1000 & $\mathrm{Gpm}$ \\
Well depth & 6400 & $\mathrm{Ft}$ \\
Cutting density & 20.6 & $\mathrm{Ppg}$ \\
Cutting diameter & 0.25 & $\mathrm{Inch}$ \\
Mud weight & 10.0 & Ppg \\
\hline
\end{tabular}




\section{Cutting carrying capacity efficiency}

Power law model and modified power law model equations were used in the calculation of the cutting carrying capacity of the proposed viscosifier additives. The following equations were used in the analysis.

$n=3.32 \log \left(\frac{\theta_{600}}{\theta_{300}}\right)$

$P_{\mathrm{v}}=\theta_{600}-\theta_{300}$

$Y_{\mathrm{p}}=\theta_{600}-P_{\mathrm{v}}$

$K=\frac{\theta_{300}}{511^{n}}$

$Y=K(y)^{n}$

$y=($ shear rate $)=\mathrm{RPM} \times 1.703$

$V_{\mathrm{ann}}=\frac{24.5 \times Q}{\mathrm{dh}^{2}-\mathrm{dp}^{2}}$

$U_{\text {eff }}=100 K\left(\frac{144 V_{\mathrm{a}}}{\mathrm{dh}-\mathrm{dp}}\right)^{n-1}$

$V_{\mathrm{s}}=12\left(\frac{U_{\mathrm{eff}}}{d \times \rho_{f}}\right)\left[\sqrt{1+\left[7.27 d\left(\frac{\rho_{p}}{\rho_{f}}-1\right)\left(\frac{d \times \rho_{f}}{U_{\mathrm{eff}}}\right)^{2}\right]}\right]-1$

(9)
$N_{\mathrm{RE}}=\frac{d \times V_{\mathrm{s}} \times \rho_{f}}{U_{\mathrm{eff}}}$

$v_{\mathrm{t}}=v_{\mathrm{a}}-v_{\mathrm{s}}$

$T_{\text {eff }}=\left(\frac{V_{\mathrm{t}}}{V_{\mathrm{a}}}\right) \times 100$

\section{Result presentation}

\section{Discussion of result}

\section{Effect of rheology on wellbore cleaning}

The rheology effect on wellbore cleaning to determine the cutting carrying capacity of the drilling fluid was evaluated in this study. Several key parameters were considered to determine the effect of rheology on wellbore cleaning. Parameters used in this study are flow behavior index $(n)$, shear stress, shear rate plastic viscosity $\left(P_{\mathrm{v}}\right)$, yield point $\left(Y_{\mathrm{p}}\right)$, yield point to plastic viscosity ratio and laminar flow consistency factor $(K)$.

Tables 10, 11, 12 and 13 show the result of the power law model to determine the cutting carrying capacity. The result showed that the yield point is high. Yield point is one of the major rheological parameters that improves the cutting carrying capacity of the fluid. Drilling fluids with high yield point have optimum wellbore cleaning capability. From Tables 14, 15, 16, 17 and 18, it can be observed that the yield
Table 10 Power law index for $5 \mathrm{~g}$

Table 11 Power law index for $8 \mathrm{~g}$

Table 12 Power law index for $10 \mathrm{~g}$

\begin{tabular}{lllllll}
\hline Samples & P.V & $Y_{\mathrm{p}}$ & RPM 600 & RPM 300 & $n$ & $K$ \\
\hline A & 28.0 & 35.0 & 63.0 & 35.0 & 0.85 & 0.17 \\
B & 27.0 & 36.0 & 63.0 & 36.0 & 0.81 & 0.23 \\
C & 28.0 & 34.0 & 62.0 & 34.0 & 0.86 & 0.16 \\
\hline
\end{tabular}

\begin{tabular}{lllllll}
\hline Samples & P.V & $Y_{\mathrm{p}}$ & RPM 600 & RPM 300 & $n$ & $K$ \\
\hline A & 29.0 & 37.0 & 66.0 & 37.0 & 0.83 & 0.21 \\
B & 29.0 & 38.0 & 67.0 & 38.0 & 0.82 & 0.23 \\
C & 26.5 & 36.0 & 62.5 & 36.0 & 0.80 & 0.25 \\
\hline
\end{tabular}

\begin{tabular}{lllllll}
\hline Samples & P.V & $Y_{\mathrm{p}}$ & RPM 600 & RPM 300 & $N$ & $K$ \\
\hline A & 27.0 & 37.0 & 64.0 & 37.0 & 0.79 & 0.27 \\
B & 26.0 & 36.0 & 62.0 & 36.0 & 0.78 & 0.28 \\
C & 27.0 & 39.0 & 66.0 & 39.0 & 0.76 & 0.27 \\
\hline
\end{tabular}


Table 13 Power law index for $15 \mathrm{~g}$

Table 14 Rheology property of the proposed viscosifier at $5 \mathrm{~g}$

\begin{tabular}{lllllll}
\hline Samples & P.V & $Y_{\mathrm{p}}$ & RPM 600 & RPM 300 & $N$ & $K$ \\
\hline A & 25.0 & 38.0 & 63.0 & 38.0 & 0.73 & 0.40 \\
B & 26.0 & 36.0 & 62.0 & 36.0 & 0.78 & 0.28 \\
C & 24.0 & 37.0 & 61.0 & 37.0 & 0.72 & 0.42 \\
\hline
\end{tabular}

\begin{tabular}{|c|c|c|c|c|c|c|}
\hline Samples & P.V & $Y_{\mathrm{p}}$ & RPM & Shear rate & Shear stress & $Y_{\mathrm{p}} / P_{\mathrm{v}}$ \\
\hline \multirow[t]{6}{*}{ A } & \multirow[t]{6}{*}{28.0} & \multirow[t]{6}{*}{36.0} & 600.0 & 1022.0 & 61.4 & \multirow[t]{6}{*}{1.3} \\
\hline & & & 300.0 & 511.0 & 36.0 & \\
\hline & & & 200.0 & 341.0 & 24.2 & \\
\hline & & & 100.0 & 170.3 & 13.4 & \\
\hline & & & 60.0 & 102.2 & 8.7 & \\
\hline & & & 30.0 & 51.1 & 4.8 & \\
\hline \multirow[t]{6}{*}{ B } & \multirow[t]{6}{*}{27.0} & \multirow[t]{6}{*}{35.0} & 600.0 & 1022.0 & 63.0 & \multirow[t]{6}{*}{1.3} \\
\hline & & & 300.0 & 511.0 & 36.0 & \\
\hline & & & 200.0 & 341.0 & 25.9 & \\
\hline & & & 100.0 & 170.3 & 14.8 & \\
\hline & & & 60.0 & 102.2 & 9.8 & \\
\hline & & & 30.0 & 51.1 & 5.6 & \\
\hline \multirow[t]{6}{*}{$\mathrm{C}$} & \multirow[t]{6}{*}{28.0} & \multirow[t]{6}{*}{34.0} & 600.0 & 1022.0 & 62.0 & \multirow[t]{6}{*}{1.2} \\
\hline & & & 300.0 & 511.0 & 34.1 & \\
\hline & & & 200.0 & 341.0 & 24.1 & \\
\hline & & & 100.0 & 170.3 & 13.3 & \\
\hline & & & 60.0 & 102.2 & 8.6 & \\
\hline & & & 30.0 & 51.1 & 4.7 & \\
\hline
\end{tabular}

Table 15 Rheology property of the proposed viscosifier at $8 \mathrm{~g}$

\begin{tabular}{|c|c|c|c|c|c|c|}
\hline Samples & P.V & $Y_{\mathrm{p}}$ & RPM & Shear rate & Shear stress & $Y_{\mathrm{p}} / P_{\mathrm{v}}$ \\
\hline \multirow[t]{6}{*}{ A } & \multirow[t]{6}{*}{29.0} & \multirow[t]{6}{*}{37.0} & 600.0 & 1022.0 & 66.1 & \multirow[t]{6}{*}{1.3} \\
\hline & & & 300.0 & 511.0 & 37.2 & \\
\hline & & & 200.0 & 341.0 & 26.6 & \\
\hline & & & 100.0 & 170.3 & 14.9 & \\
\hline & & & 60.0 & 102.2 & 10.2 & \\
\hline & & & 30.0 & 51.1 & 5.8 & \\
\hline \multirow[t]{6}{*}{ B } & \multirow[t]{6}{*}{29.0} & \multirow[t]{6}{*}{38.0} & 600.0 & 1022.0 & 67.5 & \multirow[t]{6}{*}{1.3} \\
\hline & & & 300.0 & 511.0 & 38.3 & \\
\hline & & & 200.0 & 341.0 & 27.5 & \\
\hline & & & 100.0 & 170.3 & 15.5 & \\
\hline & & & 60.0 & 102.2 & 10.2 & \\
\hline & & & 30.0 & 51.1 & 5.8 & \\
\hline \multirow[t]{6}{*}{$\mathrm{C}$} & \multirow[t]{6}{*}{26.5} & \multirow[t]{6}{*}{36.0} & 600.0 & 1022.0 & 63.9 & \multirow[t]{6}{*}{1.4} \\
\hline & & & 300.0 & 511.0 & 36.7 & \\
\hline & & & 200.0 & 341.0 & 26.6 & \\
\hline & & & 100.0 & 170.3 & 15.2 & \\
\hline & & & 60.0 & 102.2 & 10.1 & \\
\hline & & & 30.0 & 51.1 & 5.8 & \\
\hline
\end{tabular}


Table 16 Rheology property of the proposed viscosifier at $10 \mathrm{~g}$

\begin{tabular}{|c|c|c|c|c|c|c|}
\hline Samples & P.V & $Y_{\mathrm{p}}$ & RPM & Shear rate & Shear stress & $Y_{\mathrm{p}} / P_{\mathrm{v}}$ \\
\hline \multirow[t]{6}{*}{ A } & \multirow[t]{6}{*}{27.0} & \multirow[t]{6}{*}{37.0} & 600.0 & 1022.0 & 64.4 & \multirow[t]{6}{*}{1.4} \\
\hline & & & 300.0 & 511.0 & 32.2 & \\
\hline & & & 200.0 & 341.0 & 27.1 & \\
\hline & & & 100.0 & 170.3 & 15.6 & \\
\hline & & & 60.0 & 102.2 & 10.4 & \\
\hline & & & 30.0 & 51.1 & 6.0 & \\
\hline \multirow[t]{6}{*}{ B } & \multirow[t]{6}{*}{26.0} & \multirow[t]{6}{*}{36.0} & 600.0 & 1022.0 & 62.3 & \multirow[t]{6}{*}{1.4} \\
\hline & & & 300.0 & 511.0 & 36.3 & \\
\hline & & & 200.0 & 341.0 & 26.5 & \\
\hline & & & 100.0 & 170.3 & 15.4 & \\
\hline & & & 60.0 & 102.2 & 10.3 & \\
\hline & & & 30.0 & 51.1 & 6.0 & \\
\hline \multirow[t]{6}{*}{$\mathrm{C}$} & \multirow[t]{6}{*}{27.0} & \multirow[t]{6}{*}{39.0} & 600.0 & 1022.0 & 62.3 & \multirow[t]{6}{*}{1.4} \\
\hline & & & 300.0 & 511.0 & 30.9 & \\
\hline & & & 200.0 & 341.0 & 22.7 & \\
\hline & & & 100.0 & 170.3 & 13.4 & \\
\hline & & & 60.0 & 102.2 & 9.1 & \\
\hline & & & 30.0 & 51.1 & 5.4 & \\
\hline
\end{tabular}

Table 17 Rheology property of the proposed viscosifier at $15 \mathrm{~g}$

\begin{tabular}{|c|c|c|c|c|c|c|}
\hline Samples & P.V & $Y_{\mathrm{p}}$ & RPM & Shear rate & Shear stress & $Y_{\mathrm{p}} / P_{\mathrm{v}}$ \\
\hline \multirow[t]{6}{*}{ A } & \multirow[t]{6}{*}{25.0} & \multirow[t]{6}{*}{38.0} & 600.0 & 1022.0 & 62.9 & \multirow[t]{6}{*}{1.5} \\
\hline & & & 300.0 & 511.0 & 37.9 & \\
\hline & & & 200.0 & 341.0 & 28.2 & \\
\hline & & & 100.0 & 170.3 & 17.0 & \\
\hline & & & 60.0 & 102.2 & 11.7 & \\
\hline & & & 30.0 & 51.1 & 7.1 & \\
\hline \multirow[t]{6}{*}{ B } & \multirow[t]{6}{*}{26.0} & \multirow[t]{6}{*}{36.0} & 600.0 & 1022.0 & 62.3 & \multirow[t]{6}{*}{1.4} \\
\hline & & & 300.0 & 511.0 & 36.3 & \\
\hline & & & 200.0 & 341.0 & 26.5 & \\
\hline & & & 100.0 & 170.3 & 15.4 & \\
\hline & & & 60.0 & 102.2 & 10.3 & \\
\hline & & & 30.0 & 51.1 & 6.0 & \\
\hline \multirow[t]{6}{*}{$\mathrm{C}$} & \multirow[t]{6}{*}{24.0} & \multirow[t]{6}{*}{37.0} & 600.0 & 1022.0 & 61.7 & \multirow[t]{6}{*}{1.5} \\
\hline & & & 300.0 & 511.0 & 37.4 & \\
\hline & & & 200.0 & 341.0 & 26.5 & \\
\hline & & & 100.0 & 107.3 & 12.2 & \\
\hline & & & 60.0 & 102.2 & 11.8 & \\
\hline & & & 30.0 & 51.1 & 7.1 & \\
\hline
\end{tabular}

Table 18 Evaluation of $V_{\mathrm{a}}, U_{\text {eff }}$, $V_{\mathrm{s}}$ and T.E using $5 \mathrm{~g}$

\begin{tabular}{lllllllll}
\hline Samples & Dc & $N$ & $k$ & $V_{\text {a }}$ & $U_{\text {eff }}$ & $V_{\text {s }}$ & Vt & T.E $\%$ \\
\hline A & 0.25 & 0.85 & 0.17 & 106.06 & 5.74 & 4.66 & 101.40 & 96.0 \\
B & 0.25 & 0.81 & 0.23 & 106.06 & 6.25 & 4.34 & 101.72 & 96.0 \\
C & 0.25 & 0.86 & 0.16 & 106.06 & 5.81 & 4.63 & 101.43 & 96.0 \\
\hline
\end{tabular}


Table 19 Evaluation of $V_{\mathrm{a}}, U_{\text {eff }}$, $V_{\mathrm{s}}$ and T.E using $8 \mathrm{~g}$

\begin{tabular}{lllllllll}
\hline Samples & Dc & $N$ & $k$ & $V_{\text {a }}$ & $U_{\text {eff }}$ & $V_{\text {s }}$ & Vt & T.E $\%$ \\
\hline A & 0.25 & 0.83 & 0.21 & 106.06 & 6.14 & 4.39 & 101.67 & 96.0 \\
B & 0.25 & 0.82 & 0.23 & 106.06 & 6.25 & 4.34 & 101.72 & 96.0 \\
C & 0.25 & 0.80 & 0.25 & 106.06 & 5.88 & 4.57 & 101.49 & 96.0 \\
\hline
\end{tabular}

Table 20 Evaluation of $V_{\mathrm{a}}, U_{\text {eff }}$, $V_{\mathrm{s}}$ and T.E using $10 \mathrm{~g}$

\begin{tabular}{lllllllll}
\hline Samples & Dc & $N$ & $k$ & $V_{\mathrm{a}}$ & $U_{\text {eff }}$ & $V_{\mathrm{s}}$ & $\mathrm{Vt}$ & T.E $\%$ \\
\hline A & 0.25 & 0.79 & 0.27 & 106.06 & 5.91 & 4.59 & 101.47 & 96.0 \\
B & 0.25 & 0.78 & 0.28 & 106.06 & 5.70 & 4.66 & 101.40 & 96.0 \\
C & 0.25 & 0.76 & 0.27 & 106.06 & 4.75 & 5.40 & 100.66 & 95.0 \\
\hline
\end{tabular}

Table 21 Evaluation of $V_{\mathrm{a}}, U_{\text {eff }}$, $V_{\mathrm{s}}$ and T.E using $15 \mathrm{~g}$

\begin{tabular}{lllllllll}
\hline Samples & Dc & $N$ & $k$ & $V_{\mathrm{a}}$ & $U_{\text {eff }}$ & $V_{\mathrm{s}}$ & $\mathrm{Vt}$ & T.E $\%$ \\
\hline A & 0.25 & 0.73 & 0.40 & 106.06 & 5.67 & 4.76 & 101.30 & 96.0 \\
B & 0.25 & 0.78 & 0.28 & 106.06 & 5.70 & 4.66 & 101.30 & 96.0 \\
C & 0.25 & 0.72 & 0.42 & 106.06 & 5.54 & 4.76 & 101.30 & 95.0 \\
\hline
\end{tabular}

point is higher in Sample A (control sample) for 5 and $15 \mathrm{~g}$ followed by Samples B and C.

However, drilling fluids with flow behavior index $(n)$ and flow consistency factor $(K)$ values have high degree of shear thinning and good for wellbore cleaning. The flow behavior index $(n)$ ranges from 0.72 to 0.83 which suggests that the fluid is shear thinning. In addition, if $n$ is less than 1 , the fluid exhibits shear thinning. Furthermore, if $n$ is 1 , the fluid is a Newtonian fluid and $n$ values greater than 1 show shear thickening fluid. In this study, it can be observed that the values of $n$ for both the proposed local viscosifiers (Samples $\mathrm{B}$ and $\mathrm{C}$ ) materials and conventional polymers are less than 1; this suggests that the fluid is shear thinning. However, shear thinning is the decrease in viscosity with increase in shear rate.

\section{Transport cutting efficiency}

Cutting carrying capacity is important for every drilling operations. Drilling fluid must exhibit good hole cleaning. Drilled cuttings transport efficiency was used for effective hole cleaning. However, when the percentage of the transport efficiency of drilled cuttings is higher than 85 percent, the wellbore is said to be cleaned. In Tables 18, 19, 20 and 21 , it was observed that Sample B has transport efficiency of $96 \%$ for 5, 8, 10 and $15 \mathrm{~g}$, while Sample C has $96 \%$ for 5 and $8 \mathrm{~g}$ and $95 \%$ for 10 and $15 \mathrm{~g}$. It was also noted that Sample A has the same transport efficiency with Sample B. This means that the proposed viscosifiers can be used as a substitute to conventional one PAC-R. Figures 6, 7, 8 and 9 show the relationship between the shear stress and

\section{A graph of shear stress against shear rate for $5 g$}

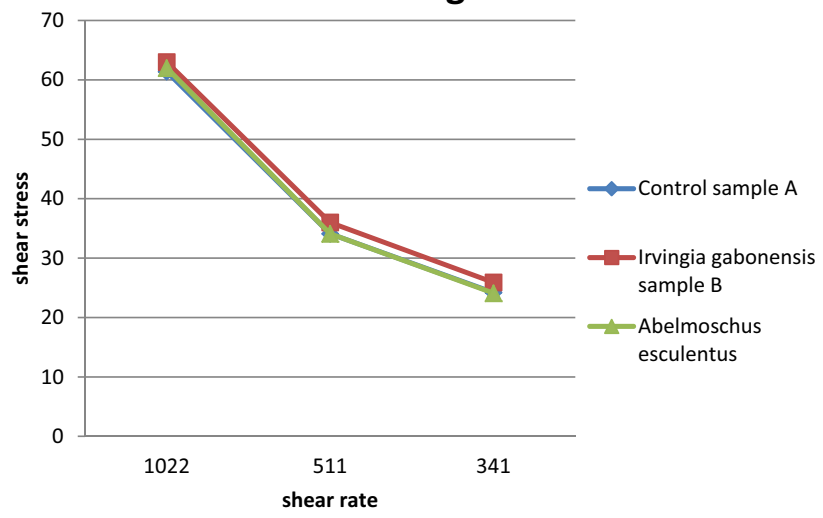

Fig. 6 Graph of shear stress against shear rate for 5 gram

the shear rate. It was observed that the proposed sample compared favorably with the control sample as showed in the figures. In addition, the water-based mud samples formulated with locally sourced materials and the conventional one PAC-R exhibits the same flow pattern. In Tables 18, 19, 20 and 21, at concentration of $2 \mathrm{~g}, 8 \mathrm{~g}, 10 \mathrm{~g}$ and $15 \mathrm{~g}$, the transport efficiency gave a higher cutting capacity for Sample B, followed by Sample C. Finally, Sample B Ogbono (Irvingia gabonensis) gave a good cutting carrying capacity followed by Sample C Okro (Abelmoschus esculentus) in comparison with the conventional one PAC-R. Based on the result of this study, the proposed viscosifiers can be suitable for effective hole cleaning. 
A graph of shear stress against shear rate for $8 \mathrm{~g}$

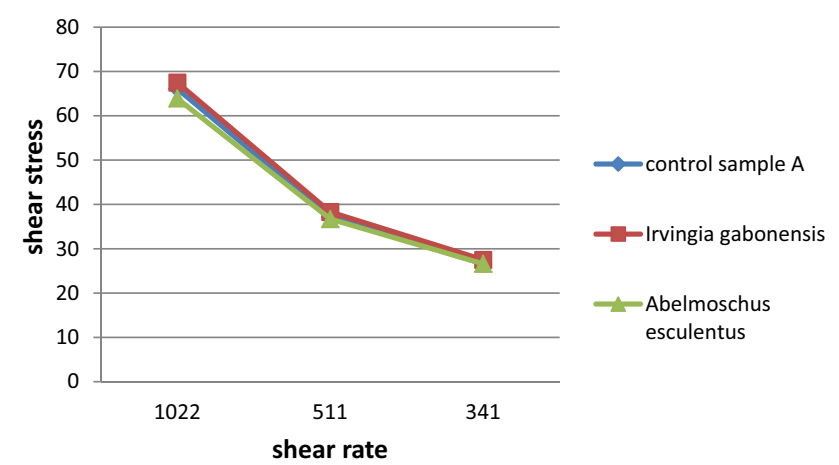

Fig. 7 Graph of shear stress against shear rate for 8 gram

\section{A graph of shear stress against shear rate for $10 \mathrm{~g}$}

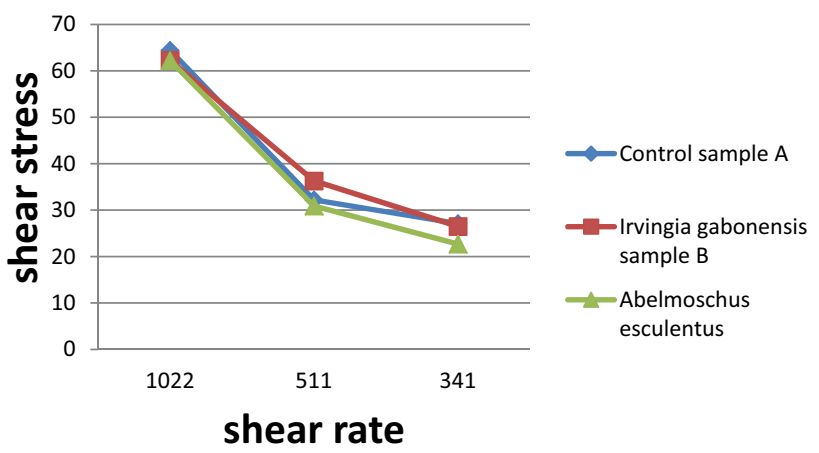

Fig. 8 Graph of shear stress against shear rate for $10 \mathrm{~g}$

\section{A graph of shear stress against shear rate for $15 \mathrm{~g}$}

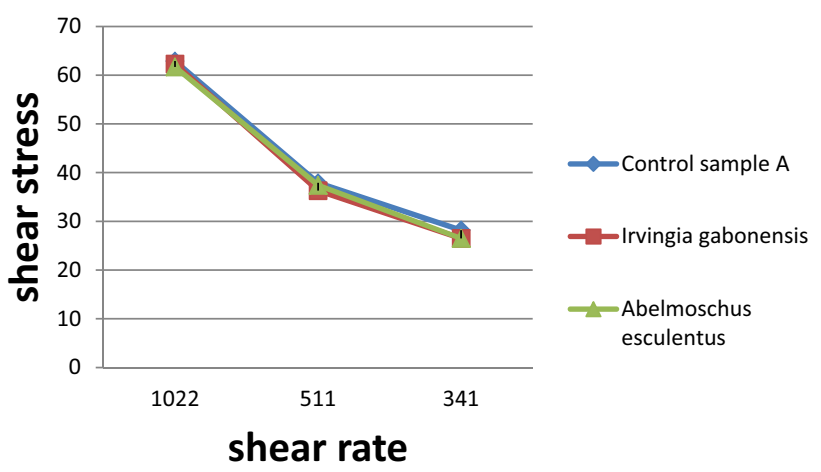

Fig. 9 Graph of shear stress against shear rate for $15 \mathrm{~g}$

\section{Characteristic of the fluid}

From the experimental work conducted on this study, it was observed that flow behavior index $(n)$ was less than one (1) for all the cases of 5, 8, 10 and $15 \mathrm{~g}$ considered in this study meaning that it is a non-Newtonian fluid exhibiting a sufficient shear thinning capacity.

\section{Conclusion}

The following conclusions can be drawn from this research study:

(i) The local viscosifiers Irvingia gabonensis (Ogbono) and Abelmoschus esculentus (Okro) formulated mud were suitable for hole drilling because of the temperature range of $150-250^{\circ} \mathrm{F}$ used in this study.

(ii) The local viscosifiers had higher yield stress, annular velocity and low slip velocity.

(iii) The proposed viscosifiers have higher transport efficiency especially Sample B (Irvingia gabonensis) followed by Sample C (Abelmoschus esculentus) which was an indication of good cutting carrying capacity.

(iv) Higher transport efficiency gave better bottom hole cleaning during drilling operations which was also dependent on cutting diameter, annular velocity and the rheology of the fluid.

(v) Modified power law model was used in the study for the evaluation of the slip velocity and annular velocity to show the effect of rheology on wellbore cleaning improvement

\section{Contribution to knowledge}

The use of local viscosifiers (Irvingia gabonensis) and (Abelmoschus esculentus) formulated in water-based drilling mud additives at different rheology, concentrations and temperatures was established in this study to show the effectiveness of the proposed viscosifiers on cutting carrying capacity and hole cleaning.

Open Access This article is distributed under the terms of the Creative Commons Attribution 4.0 International License (http://creativeco mmons.org/licenses/by/4.0/), which permits unrestricted use, distribution, and reproduction in any medium, provided you give appropriate credit to the original author(s) and the source, provide a link to the Creative Commons license, and indicate if changes were made.

\section{References}

Baroid Drilling Fluids (1998) Baroid drilling fluids handbook Baroid Drilling Fluids (2006) Baroid fluid services handbook 
Bazarnova NG, Chubik PS, Khmel'nitskii AG, Galochkin AI, Markin VI (2001) Carboxymethylated wood as a chemical reagent for preparation of drilling fluids. Russ J Appl Chem 74(4):681-686

Belavadi MN, Chukwu GA (1994). Experimental study of the parameters affecting cutting transportation in a vertical wellbore annulus. In: SPE western regional meeting, SPE Inc. Long Beach, California

Blkoor SO, Fattah KA (2013) The Influence of XC-polymer on drilling fluid filter cake properties and formation damage. J Petrol Environ Biotechnol 4:157. https://doi.org/10.4172/2157-7463.1000157

Doan QT, Oguztoreli M, Masuda Y, Yonezawa T, Koboyanshi A, Naganawa S, Kamp A (2000) Modeling of transient cuttings transport in underbalanced drilling. In: SPE 62742, IADC/SPE Asia pacific drilling technology, Kuala Lumpur, Malaysia, September, 11-13

Dosunmu A, Cosmas O, Chimaroke A, Ekehinde E (2012) Optimization of hole cleaning using dynamic real-time cuttings monitoring tools. SPE 178373, NAICE

Duru UI, Chukwu GA, Obah B (2005) Hydraulic evaluation of drilling fluid performance on hole cleaning for different rheological models. In: Paper SPE 98792 presented at 29th annual SPE international technical conference and exhibition in Abuja, Nigeria

Evelyn BE, Emmanuel EO, Adewale D, Sunny I (2019) Optimizing aqueous drilling mud system viscosity with green additives. J Pet Explor Prod Technol 9(1):315-318

Hossain ME, Wajheeuddin M (2016) The use of grass as an environmentally friendly additive in water-based drilling fluids. J Pept Sci 13(2):292-303. https://doi.org/10.1007/s12182-016-0083-8

Igwilo KC, Zaka B (2014) Evaluation of Rheological Properties of Detarium microcarpum, Brachystegia eurycoma using HerschelBuckley model and their commercial availability. J Pet Gas Eng 5(2):24-31

Igwilo KC, Anawe PAL, Okolie STA, Uzorchukwu I, Charles O (2016) Evaluation of the fluid loss property of Annona muricata and Carica papaya. Open J Yangtze Gas Oil 2:144-150. https://doi. org/10.4236/ojogas.2017.23010

Izuwa NC (2015) Evaluating the impact of rheological properties of local viscosifier on hole cleaning. FUTO J Ser (FUTOJNLS) $1(1): 67-77$
Kerunwa A, Gbaranbiri BA (2018) Evaluation of local viscosifiers as an alternative to conventional Pac-R. Adv Pet Explor Dev 15(1): $1-8$

Mozaffari S, Tchoukov P, Atias J, Czarnecki J, Nazemifard N (2015) Effect of asphaltene aggregation on rheological properties of diluted athabasca bitumen. Energy Fuels 29(9):5595-5599

Noah AZ (2013) Optimizing drilling fluid properties and flow rates for effective hole cleaning at high angle and horizontal wells. J Appl Sci Res 9(1):705-715

Ogunrinde JO, Dosunmu A (2012).Hydraulics optimization for efficient hole cleaning in deviated and horizontal wells. In: Paper SPE 162970 presented at Nigeria annual international conference and exhibition in Lagos, Nigeria

Ozbayoglu ME (2007). Estimating critical velocity to prevent bed development for horizontal-inclined wellbore. In: SPE/IADC middle east drilling technology conference and exhibition, Cairo, Egypt

Sifferman TR, Becker TE (1990) Hole cleaning in full scale inclined wellbore. In: SPE 20422, annual technical conference and exhibition, New Orleans Louisiana, September, 23-26

Unegbu CT (2010) Hole cleaning and hydraulics (Master's thesis).University of Stavanger, Faculty of Science and Technology, Norway

Walker S, Li J (2001) The effect of particle size fluid rheology and pipe eccentricity on cuttings transport. In: Presented at the SPE/ICOTA coiled tubing roundtable 2000, pp 1-8

Zhang Y, Ghaly AE, Li B (2012) Physical properties of corn residues. Am J Biochem Biotechnol 8(2):44-53. https://doi.org/10.3844/ ajbb.2012.44.53

Publisher's Note Springer Nature remains neutral with regard to jurisdictional claims in published maps and institutional affiliations. 